\title{
Disponibilidad y promoción de alcohol según la tipología de los locales y las condiciones socioeconómicas del área
}

\author{
Availability and promotion of alcohol across different outlet \\ typologies and under different area-level socioeconomic status
}

\author{
Andrea Pastor $(\mathrm{MSc}) *$, Albert Espelt $(\mathrm{PhD}) * *, * * * * * * *$, John R Villalbí (PhD)***,*****, \\ ******,*******,********, Lucía Moure $(\mathrm{PhD}) * * * * * * * * *$, Sonsoles Fuentes (PhD) $* * * * * * * * * *$, \\ Niamh Shortt $(\mathrm{PhD}) * * * * * * * * * * *$, Roberto Valiente $(\mathrm{MSC}) * * * * * * * * * * * * *$, Luisa N. Borrell

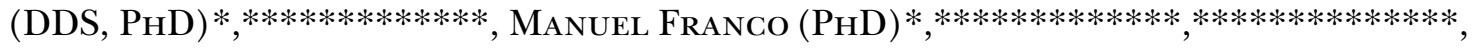 \\ XISCA SUREDA $(\mathrm{PHD}) *, * * * * * * * * * * * * *, * * * * * * * * * * * * * * *, * * * * * * * * * * * * * * * *$.
}

* Grupo de Investigación en Salud Pública y Epidemiología, Facultad de Medicina. Universidad de Alcalá, Madrid. España. ** Facultat de Ciències de la Salut de Manresa. UVicUCC, Manresa. España. *** Centro de Investigación Biomédica en Red de Epidemiología y Salud Pública (CIBERESP), Madrid. España. **** Departament de Psicobiologia i Metodologia en Ciències de la Salut. Universitat Autònoma de Barcelona (UAB), Barcelona. España. ***** Agència de Salut Pública de Barcelona, Barcelona. España. ****** Institut d'Investigació Biomèdica Sant Pau, Barcelona. España. ******* Departament de Ciències Experimentals i de la Salut. Universitat Pompeu Fabra, Barcelona. España. ******** Grupo de Trabajo sobre Alcohol. Sociedad Española de Epidemiología, Barcelona. España. ********* Departamento de Medicina Preventiva y Salud Pública. Universidade de Santiago de Compostela, Santiago de Compostela. España. ********** Dirección de Enfermedades No Transmisibles y Trauma. Salud Pública Francia. Francia. *********** Centro de Investigación sobre el Medio Ambiente, la Sociedad y la Salud. Universidad de Edimburgo, Edimburgo. Escocia. ************ Departamento de Geología, Geografía y Medio Ambiente. Universidad de Alcalá, Madrid. España. ************* Departamento de Epidemiología y Bioestadística, Escuela Graduada de Salud Publica. City University of New York, N.Y. EE. UU. ************** Facultad de Epidemiología. Universidad Johns Hopkins, Maryland. EE.UU. **************** Institut d'Investigació Biomèdica de Bellvitge-IDIBELL, Hospitalet de Llobregat. España. ***************** Consorcio de Investigación Biomédica en Enfermedades Respiratorias, CIBER en Enfermedades Respiratorias. CIBERES, Madrid. España.

\section{Resumen}

El objetivo fue caracterizar la disponibilidad y promoción de alcohol asociados a los locales de venta y consumo de alcohol en Madrid, así como explorar las diferencias en su distribución en función de la tipología del local y las características socioeconómicas del área. Se utilizó el instrumento OHCITIES para caracterizar locales situados en 42 secciones censales de Madrid durante 2016. Se registró la densidad de locales y el número de locales con amplios horarios de apertura (12 o más horas). Se registró cualquier tipo de promoción asociada al local visible desde el exterior. Se compararon los porcentajes de características de disponibilidad y promoción asociada a los locales de consumo y venta de alcohol utilizando el test de chi cuadrado y la prueba exacta de Fisher. Se estimó la densidad de disponibilidad y promoción por sección censal y se exploró su distribución en función de las características socioeconómicas del área mediante el test de Kruskal-Wallis. Se registraron 324 locales, 241 de consumo y 83 de venta. La mayoría tenía un horario amplio de apertura $(73,77 \%)$ y algún elemento promocional $(89,51 \%)$. Los locales de consumo tenían horarios más amplios de apertura y más elementos promocionales que los de venta $(p<0,001)$. Se encontraron mayor densidad de locales, amplitud de horarios y elementos promocionales en áreas de nivel socioeconómico alto (to$\operatorname{dos} p<0,001)$. La disponibilidad y promoción estuvieron asociadas con los locales de venta y consumo de alcohol en Madrid. Futuras políticas cuyo objetivo sea la prevención del consumo de alcohol deben tener en cuenta la influencia de los tipos de locales y las características socioeconómicas del área en la distribución de la disponibilidad y promoción de alcohol. Palabras clave: Disponibilidad de alcohol; locales de venta de alcohol; promoción de alcohol; nivel socioeconómico; desigualdades.

\section{Abstract}

We aimed to characterize the availability and promotion of alcohol at alcohol outlets in Madrid and to compare them according to type of outlet and area-level socioeconomic status. We used the OHCITIES instrument to characterise the alcohol outlets in 42 census tracts of Madrid in 2016. We specified alcohol availability as the density of alcohol outlets and the number of alcohol outlets with extended opening hours (12 or more). We registered any type of promotion associated to alcohol outlets that could be perceived from outside the outlet. We calculated and compared proportions of availability and promotion by alcohol outlet (on- and off-premise) using chi-squared and Fisher Exact tests. We estimated the availability and promotion of alcohol densities per census tract according to area-level socioeconomic status. To assess statistical significance, we used Kruskal-Wallis tests. We recorded 324 alcohol outlets, 241 on-premise and 83 off-premise. Most of the outlets had extended opening hours $(73.77 \%)$ and at least one sign promoting alcohol $(89.51 \%)$. More on-premise outlets had extended opening hours and higher presence of alcohol promotion than off-premise $(p<0.001)$. Higher density of alcohol outlets, extended opening hours and presence of alcohol promotion were found in higher socioeconomic areas (all $p<0.001)$. These results were also observed for on-premise alcohol outlets. Alcohol availability and promotion were associated with alcohol outlets in Madrid. Future alcohol policies regulating the availability and promotion of alcohol should consider outlet types and area-level socioeconomic status.

Key Words: Alcohol availability; alcohol outlet; alcohol promotion; socioeconomic status; inequalities.

Recibido: Julio 2019; Aceptado: Noviembre 2019.

Enviar correspondencia a: Xisca Sureda Llull, BPharm, MPH, PhD.

Universidad de Alcalá. Crta. de Madrid-Barcelona, Km. 33.600. Alcalá de Henares, Madrid, 28871, España. Tel: +34 918854573.

E-mail: francisca.sureda@uah.es 
$\mathrm{E}$ 1 alcohol es uno de los principales factores asociados a la discapacidad y muerte a nivel global (Organización Mundial de la Salud, 2018). El uso nocivo de alcohol se ha asociado con efectos adversos para la salud (Galán, Valencia-Martín, Guallar-Castillón y Rodríguez-Artalejo, 2014; Griswold et al., 2018), desventajas socioeconómicas (Waller y Iritani, 2013) y problemas sociales (Cunradi, 2010; Mair, Gruenewald, Ponicki y Remer, 2013). A nivel mundial, los problemas relacionados con el alcohol han aumentado en los últimos veinte años (Organización Mundial de la Salud, 2018).

En España, el consumo de alcohol está considerado como parte de su cultura (Sureda, Villalbí, Espelt y Franco, 2017a). Aunque ha disminuido la prevalencia del consumo diario excesivo de alcohol y de la mortalidad atribuible al alcohol, las tasas de binge drinking se mantuvieron elevadas en hombres entre 25-29 años (30\%) y mujeres entre 20-24 años $(20 \%)$, según datos del 2017 (Plan Nacional sobre Drogas, 2017).

Según estudios previos, las características del entorno físico como la disponibilidad y promoción de alcohol podrían afectar al consumo del mismo (Bryden, Roberts, Petticrew y McKee, 2013; Sureda et al., 2018a). La disponibilidad de alcohol se describe normalmente como la densidad de o la proximidad a locales de venta y/o consumo de alcohol (Popova, Giesbrecht, Bekmuradov y Patra, 2009). Algunos estudios han mostrado asociaciones positivas entre la disponibilidad de locales venta y/o consumo de alcohol y el consumo de alcohol (Sherk et al., 2018). Por ejemplo, un estudio en Australia en adolescentes halló un aumento de $17 \%$ en el consumo de alcohol al aumentar un $10 \%$ la densidad de locales de venta y/o consumo de alcohol (Rowland et al., 2016). Normalmente se distingue entre aquellos locales en los que se puede consumir alcohol (como bares y restaurantes) y aquellos locales en los se puede comprar alcohol pero no consumirlo (como supermercados o tiendas de conveniencia) (Rowland et al., 2014; Shortt et al., 2015). Los estudios que han comparado la disponibilidad de alcohol según el tipo de local (de venta o de consumo) encontraron que hubo efectos diferentes sobre las conductas relacionadas con el alcohol (Giesbrecht et al., 2015; Young, Macdonald y Ellaway, 2013). Por ejemplo, un estudio que analizó las asociaciones entre la disponibilidad de locales de venta y consumo de alcohol sobre el consumo de alcohol en adolescentes halló un aumento del riesgo del 5,30\% por cada aumento del $10 \%$ en la densidad de locales de venta, pero un aumento de solo del $1,68 \%$ para los locales de consumo (Rowland et al., 2014). Otros estudios también han encontrado asociaciones más fuertes entre los patrones de consumo de riesgo, como el binge drinking, en jóvenes y la disponibilidad de locales de venta que en relación con la disponibilidad de locales de consumo (Halonen et al., 2013; Young et al., 2013).
De manera similar, una elevada disponibilidad de locales de venta y/o consumo de alcohol facilita las oportunidades de promoción del mismo (Bryden et al., 2013; Sureda et al., 2017b). Estudios previos han explorado sobre todo el papel de la publicidad y patrocinio de alcohol (Anderson, De Bruijn, Angus, Gordon y Hastings, 2009a; Westberg, Stavros, Smith, Munro y Argus, 2018). No obstante, han ignorado otros elementos promocionales habitualmente ubicados en los locales de venta y/o consumo. Por tanto, la literatura existente puede haber subestimado la promoción del alcohol. A pesar de ésta subestimación de la exposición a la promoción de alcohol, estudios anteriores han encontrado asociación entre la promoción y ciertos patrones de consumo de riesgo de alcohol, especialmente entre niños y jóvenes (Bosque-Prous et al., 2014; Esser, Waters, Smart y Jernigan, 2016).

Además de las tipologías de local, las características socioeconómicas (NSE) del área pueden afectar la distribución de la disponibilidad y promoción de alcohol en la ciudad (Bryden et al., 2013; Morrison, Gruenewald y Ponicki, 2015). La evidencia sugiere mayor disponibilidad de locales de venta y/o consumo de alcohol en áreas de NSE bajo que en áreas de NSE alto (Hay, Whigham, Kypri y Langley, 2009; Major et al., 2014; Sudhinaraset, Wigglesworth y Takeuchi, 2015). No obstante, está relación es menos clara cuando se consideran las distintas tipologías de locales (Angus et al., 2017; Rhew, Kosterman y Lee, 2017). La distribución de la promoción de alcohol según NSE se ha explorado menos. No obstante, algunos estudios han encontrado mayor promoción de alcohol en áreas más deprimidas socieconómicamente (Gentry et al., 2018; Hackbarth, Silvestri y Cosper, 1995; Lee y Callcott, 1994).

Se han usado metodologías diferentes para describir la disponibilidad y promoción de alcohol en entornos urbanos. Algunos estudios han utilizado bases de datos secundarias (Richardson, Hill, Mitchell, Pearce y Shortt, 2015; Shortt et al., 2015); otros han utilizado datos auto-reportados (Scribner, Cohen y Fisher, 2000; Wechsler, Lee, Hall, Wagenaar y Lee, 2002). No obstante, ambos métodos aportan información limitada respecto de la disponibilidad y promoción, y no están exentos de sesgos. En este estudio se utiliza el instrumento OHCITIES, basado en la observación social sistemática en la calle (estudio de campo) (Sureda et al., 2017b). Esta metodología permite definir características de los barrios a pie de calle que serían difíciles o casi imposibles de captar utilizando otras metodologías, mediante la observación directa (Costa et al., 2017; Raudenbush y Sampson, 1999). El objetivo de este estudio es caracterizar la disponibilidad y promoción de alcohol en locales de venta y consumo de alcohol en la ciudad de Madrid, España, usando este instrumento. Además, se comparan las diferencias de disponibilidad y promoción de alcohol por tipo de local de venta o de consumo de alcohol y por NSE del área. 


\section{Materiales y Métodos}

\section{Diseño del estudio y tamaño muestral}

Estudio transversal y observacional realizado en Madrid, España, en 2016. Madrid se divide en 21 distritos, a su vez subdivididos en 128 barrios y 2412 secciones censales. Este estudio usó las secciones censales. Una sección censal es el área administrativa más pequeña, con una media de población de 1500 habitantes y definida por límites fácilmente identificables.

Usamos un diseño de muestreo multietapa para seleccionar las áreas de observación y asegurar la representatividad de las características sociodemográficas de la ciudad completa. En primer lugar, se seleccionaron dos barrios mediante un diseño de muestreo no probabilístico para cada distrito (42 barrios en total) representando las siguientes características socioeconómicas: desempleo, trabajo precario, clasificación ocupacional, nivel de estudios e inmigración. En segundo lugar se seleccionó la sección censal media de cada barrio $(\mathrm{n}=42)$ según la densidad poblacional, densidad empresarial, nivel de estudios, inmigración y edades de la población. El procedimiento para elegir las secciones censales ha sido descrito previamente en otros estudios (Sureda et al., 2018b).

\section{Observación social sistemática: disponibilidad y promoción de alcohol asociada a los puntos de venta y/o consumo de alcohol}

\section{Instrumento OHCITIES y procedimiento de recogida de datos}

Usamos el instrumento OHCITIES, una herramienta válida y fiable, para caracterizar sistemáticamente los elementos relacionados con el entorno de alcohol (Sureda et al., 2017b), como la disponibilidad y promoción de alcohol. Los valores psicométricos del instrumento superaron el $80 \%$ de acuerdo para las variables de exposición al alcohol asociada tanto a los locales de venta como a los de venta y consumo de alcohol, y superaron valores de ,80 para fiabilidad inter-observador y fiabilidad test-retest (Sureda et al., 2017b). Se caracterizaron los locales de venta y/o consumo de alcohol mediante observación social sistemática en las 42 secciones censales seleccionadas.

Los locales de venta y consumo de alcohol se clasificaron en: 1) bares o similares (incluyendo cafeterías, cervecerías o bodegas (donde se sirve mayoritariamente vinos no asociados a marca); 2) restaurantes (incluyendo también restaurantes de comida para llevar y comida rápida); 3) clubs nocturnos (incluyendo pubs con música, coctelerías, clubs nocturnos o discotecas) y 4) otros tipos de locales (librerías, establecimientos de cata de vinos, etc.). Los locales de venta de alcohol se clasificaron en: 1) supermercados; 2) tiendas de conveniencia; 3) tiendas de alimentación especializadas (incluyendo fruterías, verdulerías, carnicerías, pescaderías y panaderías); y 4) tiendas de vinos y licores.
Tres observadores entrenados recogieron los datos entre mayo y noviembre 2016, en días laborables entre las 16h-21h para captar todos los locales de venta y/o consumo de alcohol abiertos. Recorrieron ambos lados de las calles ubicadas en la sección censal elegida rellenando el cuestionario OHCITIES. La ruta a seguir en cada sección censal se definió previamente en un mapa que los observadores usaron el día de la recogida de datos. Un mismo observador completó cada sección censal. Se registraron todos los locales de venta y/o consumo de alcohol en cada sección censal.

\section{Variables del entorno relacionados con el alcohol}

Las variables relacionadas con la disponibilidad de alcohol incluyeron el número absoluto de locales de venta y/o consumo de alcohol por sección censal y el número de locales de venta y/o consumo de alcohol con un horario amplio de apertura. Los horarios de apertura se detectaron en los carteles en el exterior de cada establecimiento. En el caso de establecimientos cuyos horarios no eran visibles se imputó la moda de horas en las que el local estaba abierto por sección censal para cada tipo de local (venta y/o consumo). En base a estos dos datos, se dividió esta variable en dos categorías: locales con (1) apertura de hasta 12 horas, y (2) apertura de más de 12 horas.

Para valorar la distribución de disponibilidad según tipo de local (venta o venta y consumo de alcohol), calculamos el porcentaje de locales abiertos más de 12 horas. Además, estimamos el número absoluto de locales de venta $\mathrm{y} / \mathrm{o}$ consumo de alcohol y el número de locales de venta y/o consumo de alcohol abiertos más de 12 horas por 10000 habitantes para cada sección censal para explorar la distribución según el NSE del área.

Las variables relacionadas con la promoción de alcohol incluyeron la presencia de: i) publicidad o patrocinio en los escaparates o ventanas visibles; ii) elementos estructurales, como toldos, rótulos y/o carta de bebidas asociados a una bebida, producto o marca alcohólica específica; iii) mobiliario, como barriles, cajas de alcohol, mesas, sillas, sombrillas, servilleteros o ceniceros asociados a un producto o marca de alcohol específica (esta variable sólo aplica a los locales de venta y consumo de alcohol); iv) presencia de bebidas alcohólicas (botellas de alcohol, latas, y cerveza, sidra o vino de barril/grifo) dentro del establecimiento y visibles desde el exterior; y v) presencia de botellas y/o latas de alcohol expuestas en los escaparates. Se compuso una variable de promoción general ( $\mathrm{si} / \mathrm{no}$ ) considerando la presencia de al menos un elemento promocional de los mencionados antes.

Para valorar la distribución de promoción según tipo de local (venta y/o consumo de alcohol), calculamos el porcentaje de locales con los diferentes signos de promoción. También se estimó el número de locales con al menos un signo de promoción por cada 10.000 habitantes de cada sección censal para encontrar diferencias por NSE. 


\section{Características socioeconómicas}

Usamos un índice compuesto de características socioeconómicas (Gullón et al., 2017) basado en 7 indicadores recopilados de varias bases de datos. El registro del padrón municipal de Madrid, un censo continuo y universal recopilado con fines administrativos (http://www-2.munimadrid.es/CSE6/jsps/menuBancoDatos.jsp), se usó para recoger datos de: (1) nivel bajo de estudios (el porcentaje de personas mayores de 25 años con estudios primarios o inferiores) y (2) nivel alto de estudios (el porcentaje de personas mayores de 25 años con estudios universitarios o superiores). Usamos el registro de la Seguridad Social (http:/ / www.seg-social.es/Internet_1/Estadistica/Est/index.htm) para recoger datos de: (3) empleo a tiempo parcial (porcentaje de trabajadores en empleos a jornada parcial), (4) empleo temporal (porcentaje de trabajadores en empleos temporales), (5) trabajadores manuales (porcentaje de trabajadores en empleos manuales/no cualificados). Por último, usamos el informe sobre viviendas de Idealista (https://www.idealista.com/informes-precio-vivienda), una gran empresa española del sector inmobiliario, y el registro del servicio público de empleo estatal (http://www.sepe. es/contenidos/que_es_el_sepe/estadisticas/datos_estadisticos/empleo/index.html) para obtener indicadores del (6) precio medio de viviendas (por m2) y de la (7) tasa de desempleo, respectivamente. Obtuvimos los datos para todos los indicadores a nivel de sección censal para el 2015.

Se categorizó el índice de NSE en terciles (bajo, medio, alto) en base a todas las secciones censales en Madrid.

\section{Análisis estadístico}

Se utilizaron estadísticos descriptivos para describir la disponibilidad y promoción de alcohol en el entorno, según tipo de local de venta y/o consumo de alcohol. Comparamos la disponibilidad y promoción en locales de venta y consumo de alcohol con el chi cuadrado, y en locales de venta con la prueba exacta de Fisher, debido al tamaño muestral pequeño. Se utilizó el test de Kruskal-Wallis para distribuciones no paramétricas para examinar la distribución, entre los diferentes tertiles de NSE, de las densidades relacionadas con disponibilidad y promoción de alcohol de los locales de venta y venta y consumo de alcohol. El nivel de significancia se fijó en un $95 \%$ y el software utilizado para realizar los análisis fue STATA v12.0.

\section{Resultados}

\section{Descripción de la muestra}

Observamos un total de 324 locales de venta y/o consumo de alcohol (241 de venta y consumo y 83 de venta) en las 42 secciones censales (Tabla 1). El tipo de local más común fueron cafeterías y bares $(74,38 \%)$, seguido de tiendas de conveniencia $(19,14 \%)$. Por sección censal, hubo entre 0-37 locales de venta y consumo de alcohol y entre 0-7 para locales de venta de alcohol. La mayoría de los locales de venta y consumo $(75,93 \%)$ y de venta $(85,54 \%)$ se econtraron abiertos durante la recogida de datos.

Tabla 1. Descripción de la muestra del estudio según tipo de local de venta y/o consumo de alcohol en 42 secciones censales de la ciudad de Madrid, 2016.

\begin{tabular}{cccc}
\hline $\begin{array}{c}\text { Tipo de local de venta y/o consumo de alcohol } \\
\text { en las } \mathbf{4 2} \text { secciones censales de la muestra }\end{array}$ & $\mathbf{N}$ & $\%$ \\
\hline LOCALES DE VENTA Y/O CONSUMO DE ALCOHOL & 324 & \\
\hline Locales de venta y consumo & $\mathbf{2 4 1}$ & $\mathbf{7 4 , 3 8}$ \\
Bar o similar & 176 & 54,32 \\
Restaurante & 50 & 15,43 \\
Clubs nocturnos & 13 & 4,01 \\
Otros & 2 & 0,62 \\
\hline Locales de venta & $\mathbf{8 3}$ & $\mathbf{2 5 , 6 2}$ \\
Supermercados & 11 & 3,39 \\
Tiendas de conveniencia & 62 & 19,14 \\
Tiendas especializadas & 8 & 2,47 \\
Tiendas de vinos o licores & 2 & 0,62 \\
\hline
\end{tabular}

\section{Variables relacionadas con el alcohol asociadas a los locales de venta y/o consumo de alcohol}

La Tabla 2 muestra las características de exposición relacionadas con la disponibilidad y promoción de alcohol asociadas a los locales de venta y/o consumo del mismo. En general, el 73,77\% de los locales tenía un horario amplio de apertura (más de 12 horas), y el 89,51\% tenía al menos un elemento de promoción de alcohol asociado al local.

En cuanto a los elementos relacionados con la promoción de alcohol, el 32,41\% de los locales tenía publicidad o patrocinio en los escaparates o ventanas visibles desde el exterior; el 36,73\% tenía al menos un elemento estructural asociado a un producto o marca alcohólica específica; el $10,80 \%$ tenía botellas o latas de alcohol en el escaparate; y el 50,47\% tenía expuestos más de dieciséis bebidas alcohólicas (botellas, latas o barriles/grifos) dentro del establecimiento pero visibles desde el exterior. En comparación con los locales de venta, los locales de venta y consumo de alcohol tuvieron horarios más amplios $(p<0,001)$; mayor presencia de al menos un signo de promoción de alcohol asociada al local $(p=0,001)$; y de al menos un elemento estructural asociado a un producto o marca alcohólica específica $(p<0,001)$. Hubo más locales de venta de alcohol con botellas y/o latas expuestas en el escaparate $(p=0,039)$, y de publicidad o patrocinio en los escaparates, que locales de venta y consumo. No hubo diferencia entre los locales de venta y de venta y consumo de alcohol con respecto a la presencia de bebidas alcohólicas y otros productos relacionados dentro del establecimiento y visibles desde el exterior. La Figura 1 muestra ejemplos de exposición al alcohol en locales de venta y/o consumo de alcohol. 
Tabla 2. Variables relacionadas con el alcohol asociadas a locales de venta y/o consumo de alcohol en 42 secciones censales de la ciudad de Madrid, 2016.

\begin{tabular}{|c|c|c|c|c|c|}
\hline & \multicolumn{5}{|c|}{ TIPO DE LOCAL } \\
\hline & \multicolumn{2}{|c|}{$\begin{array}{c}\text { Total } \\
(\mathrm{N}=\mathbf{3 2 4} \text { locales })\end{array}$} & \multirow{2}{*}{$\begin{array}{c}\text { Locales de venta y consumo } \\
\text { de alcohol }(\mathrm{n}=\mathbf{2 4 1})\end{array}$} & \multirow{2}{*}{$\begin{array}{l}\text { Locales de venta de alcohol } \\
\left(\mathrm{n}=\mathbf{8 3}_{3}\right)\end{array}$} & \multirow[b]{2}{*}{ Valor $p^{\star}$} \\
\hline & $\mathbf{N}$ & $\%$ & & & \\
\hline \multicolumn{6}{|l|}{ DISPONIBILIDAD } \\
\hline Horario de apertura & & & & & 0,001 \\
\hline Más de 12 horas & 239 & 73,77 & 78,84 & 59,04 & \\
\hline PROMOCIÓN & & & & & 0,001 \\
\hline Con promoción & 290 & 89,51 & 92,95 & 79,52 & \\
\hline \multicolumn{5}{|c|}{ Publicidad o patrocinio en los escaparates o ventanas visibles } & 0,053 \\
\hline Presente & 105 & 32,41 & 29,46 & 40,96 & \\
\hline \multicolumn{5}{|c|}{ Elementos estructurales asociados a bebidas alcohólicas y otros productos relacionados } & 0,001 \\
\hline Presente & 119 & 36,73 & 47,72 & 4,82 & \\
\hline Botellas y/o latas en & & & & & 0,039 \\
\hline Sí & 35 & 10,80 & 8,71 & 16,87 & \\
\hline \multicolumn{5}{|c|}{ Botellas, latas y/o grifos de alcohol dentro del establecimiento } & 0,155 \\
\hline Ninguno & 87 & 26,85 & 28,22 & 22,89 & \\
\hline Entre 1 y 15 & 67 & 20,68 & 22,41 & 15,66 & \\
\hline Más de 16 & 170 & 52,47 & 49,38 & 61,45 & \\
\hline
\end{tabular}

Nota. * Se estimó el valor $p$ con el test de chi-cuadrado entre locales de venta y consumo y locales de venta de alcohol.

Exploramos las diferencias de la exposición al alcohol según tipo de local de venta y consumo de alcohol (Tabla 3). Se excluyeron del análisis Otros tipos de locales de venta y consumo de alcohol porque únicamente se registraron dos locales en esta categoría (una biblioteca y un establecimiento de cata). Finalmente, se analizaron 239 locales de venta y consumo de alcohol. Los bares tuvieron horarios más amplios de apertura que los restaurantes y los clubs nocturnos $(p<0,001)$; tuvieron mayor presencia de al menos un signo de promoción de alcohol asociada al local $(p<0,001)$; mayor presencia de, al menos, un elemento estructural asociado a un producto o marca alcohólica específica $(p<0,001)$; mayor presencia de algún tipo de mobiliario asociado a un

a)

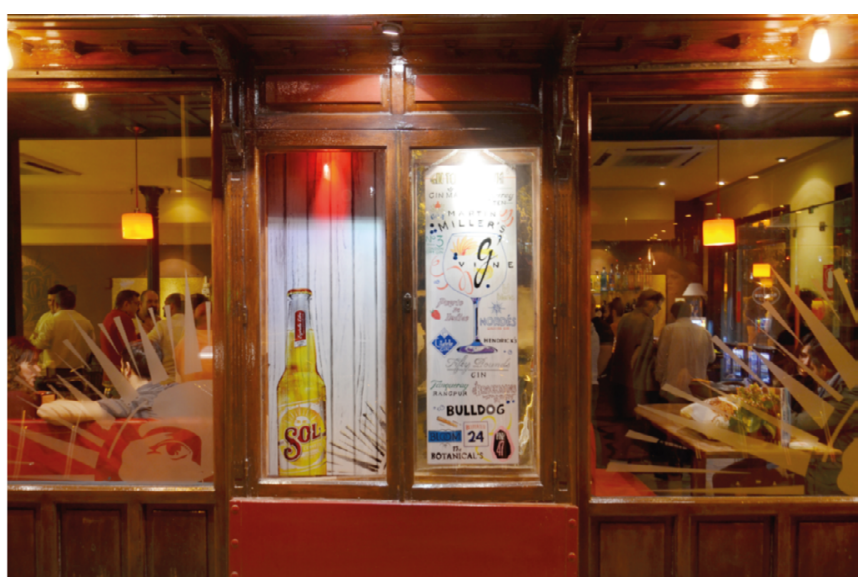

Nota. Panel a) Bar (local de venta y consumo) en el cual se promocionan diferentes tipos y marcas de bebidas alcohólicas en sus ventanas. Bebidas alcohólicas y productos relacionados (botellas y grifos de alcohol) dentro del establecimiento y visibles desde el exterior. producto o marca alcohólica específica $(p=0,003)$; y mayor número de bebidas alcohólicas y otros productos relacionados dentro del establecimiento y visibles desde el exterior $(p<0,001)$. No se encontraron diferencias significativas en la presencia de publicidad o patrocinio en los escaparates o ventanas visibles desde el exterior y la presencia de botellas y/o latas de alcohol en los escaparates según los tipos de locales de venta y consumo.

Al comparar las diferencias entre tipos de locales de venta (incluyendo supermercados y tiendas de conveniencia), no hallamos diferencias en las características de exposición al alcohol en relación a la disponibilidad y promoción de alcohol (Tabla 4).

b)

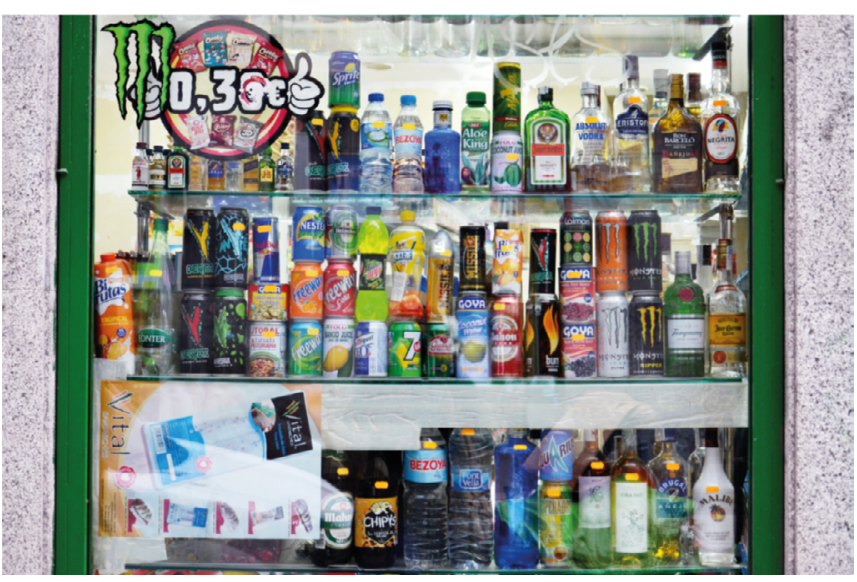

Nota. Panel b) Tienda de conveniencia (local de venta de alcohol) donde los dueños/as muestran las bebidas alcohólicas y productos relacionados en el escaparate (más de 16 bebidas alcohólicas visibles desde el exterior).

Figura 1. Ejemplos de disponibilidad y promoción asociadas a locales de venta y/o consumo de alcohol de alcohol en 42 secciones censales de la ciudad de Madrid, 2016. (Fotografías: Victor G. Carreño). 
Tabla 3. Variables relacionadas con el alcohol según tipo de local de venta y consumo de alcohol (bares, restaurantes o clubs nocturnos) en 42 secciones censales de la ciudad de Madrid, 2016.

\begin{tabular}{|c|c|c|c|c|c|c|}
\hline & \multicolumn{6}{|c|}{ LOCALES DE VENTA Y CONSUMO DE ALCOHOL } \\
\hline & \multicolumn{2}{|c|}{ Total $(\mathrm{N}=239$ locales $)$} & \multirow{2}{*}{$\begin{array}{c}\text { Bares }(\mathrm{n}=\mathbf{1 7 6}) \\
\%\end{array}$} & \multirow{2}{*}{$\frac{\text { Restaurantes }(\mathrm{n}=\mathbf{5 0})}{\%}$} & \multirow{2}{*}{$\begin{array}{c}\text { Clubs nocturnos }(n=13) \\
\%\end{array}$} & \multirow{2}{*}{ Valor $p$} \\
\hline & $\mathbf{N}$ & $\%$ & & & & \\
\hline \multicolumn{7}{|l|}{ DISPONIBILIDAD } \\
\hline \multicolumn{6}{|l|}{ Horario de apertura } & 0,001 \\
\hline Más de 12 horas & 188 & 78,66 & 88,64 & 44,00 & 76,92 & \\
\hline \multicolumn{6}{|l|}{ PROMOCIÓN } & 0,001 \\
\hline Con promoción & 222 & 92,89 & 96,59 & 90,00 & 53,85 & \\
\hline \multicolumn{6}{|c|}{ Publicidad o patrocinio en los escaparates o ventanas visibles } & 0,200 \\
\hline Presente & 71 & 29,71 & 30,68 & 32,00 & 7,69 & \\
\hline \multicolumn{6}{|c|}{ Elementos estructurales asociados a bebidas alcohólicas y otros productos relacionados } & 0,001 \\
\hline Presente & 113 & 47,28 & 54,55 & 24,00 & 38,46 & \\
\hline \multicolumn{6}{|c|}{ Mobiliario asociado a bebidas alcohólicas y otros productos relacionados } & 0,003 \\
\hline Presente & 76 & 31,80 & 37,50 & 20,00 & - & \\
\hline \multicolumn{6}{|c|}{ Botellas $y / 0$ latas en el escaparate } & 0,384 \\
\hline Sí & 21 & 8,79 & 8,52 & 12,00 & - & \\
\hline \multicolumn{6}{|c|}{ Botellas, latas y/o grifos de alcohol dentro del establecimiento } & 0,001 \\
\hline Ninguno & 68 & 28,45 & 20,45 & 40,00 & 92,31 & \\
\hline Entre 1 y 15 & 54 & 22,59 & 24,43 & 22,00 & - & \\
\hline Más de 16 & 117 & 48,95 & 55,11 & 38,00 & 7,69 & \\
\hline
\end{tabular}

Nota. ${ }^{*}$ Se estimó el valor $p$ con el test de chi-cuadrado entre bares o similar, restaurantes y clubs nocturnos para locales de venta y consumo de alcohol.

\section{Exposición al alcohol en locales de venta y/o consumo de alcohol por características socioeconómicas del área}

La densidad media de locales de venta y/o consumo de alcohol en las 42 secciones censales estudiadas fue de 56,17 locales por 10000 habitantes (incluyendo de venta y/o consumo de alcohol). Cuando exploramos su distribución por NSE del área (Tabla 5), la densidad media de locales de venta y/o consumo de alcohol por 10000 habitantes aumentó de 64,01 en áreas con un bajo NSE hasta 94,11 en áreas con un alto NSE $(p<0,001)$. Se observó un patrón similar para las demás variables de exposición al alcohol. La densidad de locales de venta y/o consumo de alcohol con un horario amplio de apertura fue más elevada en áreas con un NSE medio y alto que en áreas con un NSE bajo $(p<0,001)$. La densidad de al menos un signo de promo- ción de alcohol asociada a los locales fueron más elevada en las áreas con alto NSE $(p<0,001)$.

La densidad media de locales de venta y consumo de alcohol por 10000 habitantes fue más alta en áreas con un NSE medio y alto que en áreas más deprimidas (93,15 y 82,35 vs. 44,10 locales de venta y consumo de alcohol por 10000 habitantes, respectivamente, $p<0,001)$. Los mismos patrones se observaron para la densidad de puntos de venta y consumo de alcohol con amplios horarios de apertura y cualquier tipo de elemento promocional $(p<0,001$; Tabla 6$)$. No se observaron diferencias significativas en la distribución de los locales de venta de alcohol en función del NSE ( $p=0,776$; Tabla 7 ). No hubo tampoco diferencias en la distribución de la densidad de locales de venta con amplios horarios de apertura ni con presencia de al menos un signo de promoción de alcohol según el NSE del área ( $p=0,448$ y 0,726, respectivamente).

Tabla 4. Variables relacionadas con el alcohol según tipo de local de venta (supermercados y tiendas de conveniencia) en 42 secciones censales de la ciudad de Madrid, 2016.

\begin{tabular}{|c|c|c|c|c|c|}
\hline & \multicolumn{5}{|c|}{ LOCALES DE VENTA DE ALCOHOL } \\
\hline & \multicolumn{2}{|c|}{ Total ( $\mathrm{N}=73$ locales de venta) } & $\begin{array}{c}\begin{array}{c}\text { Supermercados } \\
(\mathbf{n}=11)\end{array} \\
\%\end{array}$ & $\begin{array}{l}\text { Tiendas de conveniencia } \\
\qquad(n=62) \\
\%\end{array}$ & Valor $p^{\star}$ \\
\hline DISPONIBILIDAD & & & & & 0,562 \\
\hline Más de 12 horas & 48 & 65,75 & 63,64 & 66,13 & \\
\hline PROMOCIÓN & & & & & 0,353 \\
\hline Presente & 59 & 80,82 & 72,73 & 82,26 & \\
\hline
\end{tabular}

Nota. * Se estimó el valor $p$ con la prueba exacta de Fisher entre supermercados y tiendas de conveniencia para locales de venta de alcohol. 
Andrea Pastor, Albert Espelt, Joan R Villalbí, Lucía Moure, Sonsoles Fuentes, Niamh Shortt,

Roberto Valiente, Luisa N. Borrell, Manuel Franco, Xisca Sureda

Tabla 5. Variables relacionadas con el alcohol por cada 10 ooo habitantes asociadas a los locales de venta y/o consumo de alcohol según características socioeconómicas (NSE) del área en 42 secciones censales de la ciudad de Madrid, 2016.

\begin{tabular}{|c|c|c|c|c|c|c|c|}
\hline & \multicolumn{7}{|c|}{ Características socioeconómicas } \\
\hline & \multicolumn{2}{|c|}{ Bajo $(n=77)$} & \multicolumn{2}{|c|}{ Medio $(n=138)$} & \multicolumn{2}{|c|}{ Alto $(n=109)$} & \multirow{2}{*}{ Valor $p^{*}$} \\
\hline & Media & Rango & Media & Rango & Media & Rango & \\
\hline $\begin{array}{l}\text { TOTAL LOCALES DE VENTA } \\
\text { Y/O CONSUMO DE ALCOHOL }\end{array}$ & 64,01 & $41,39-73,91$ & 88,44 & $51,81-319,30$ & 94,11 & $72,78-140,85$ & $<0,001$ \\
\hline \multicolumn{8}{|l|}{ DISPONIBILIDAD } \\
\hline \multicolumn{8}{|l|}{ Horario de apertura } \\
\hline Más de 12 horas & 38,58 & $25,44-62,53$ & 68,03 & $45,78-203,19$ & 60,31 & $43,67-92,20$ & $<0,001$ \\
\hline \multicolumn{8}{|l|}{ PROMOCIÓN } \\
\hline Presente & 62,54 & $22,83-66,15$ & 82,19 & $51,81-253,99$ & 94,12 & $72,78-133,43$ & $<0,001$ \\
\hline \multicolumn{8}{|c|}{ Publicidad o patrocinio en los escaparates o ventanas visibles } \\
\hline Presente & 16,54 & $16,54-17,06$ & 43,84 & $20,06-145,14$ & 30,67 & $14,56-59,30$ & $<0,001$ \\
\hline Presente & 18,28 & $17,06-33,08$ & 30,09 & $22,21-87,08$ & 32,89 & $14,56-51,89$ & $<0,001$ \\
\hline \multicolumn{8}{|c|}{ Botellas y/o latas en el escaparate } \\
\hline Sí & 0,00 & $0,00-11,03$ & 5,02 & $0,00-43,54$ & 30,67 & $7,28-37,06$ & $<0,001$ \\
\hline \multicolumn{8}{|c|}{ Botellas, latas y/o grifos de alcohol dentro del establecimiento } \\
\hline Más de 16 & 18,28 & $9,13-44,10$ & 38,36 & $31,81-181,42$ & 49,34 & $35,29-81,54$ & $\langle 0,001$ \\
\hline
\end{tabular}

Nota. * Se estimó el valor $p$ con el test de Kruskal-Wallis para los datos continuos.

Tabla 6. Variables relacionadas con el alcohol por cada 10 ooo habitantes asociadas a los locales de venta y consumo de alcohol según características socioeconómicas (NSE) del área en 42 secciones censales de la ciudad de Madrid, 2016.

\begin{tabular}{|c|c|c|c|c|c|c|c|}
\hline & \multicolumn{7}{|c|}{ Características socioeconómicas } \\
\hline & \multicolumn{2}{|c|}{ Bajo $(n=50)$} & \multicolumn{2}{|c|}{ Medio $(n=105)$} & \multicolumn{2}{|c|}{ Alto $(n=86)$} & \multirow{2}{*}{ Valor $p^{\star}$} \\
\hline & Media & Rango & Media & Rango & Media & Rango & \\
\hline $\begin{array}{l}\text { TOTAL LOCALES DE VENTA } \\
\text { Y CONSUMO DE ALCOHOL }\end{array}$ & 44,10 & $22,83-56,85$ & 93,15 & $49,65-268,51$ & 82,35 & $49,34-118,60$ & $<0,001$ \\
\hline \multicolumn{8}{|l|}{ DISPONIBILIDAD } \\
\hline \multicolumn{8}{|l|}{ Horario de apertura } \\
\hline Más de 12 horas & 34,32 & $22,83-45,48$ & 71,23 & $49,65-174,17$ & 58,82 & $32,89-92,02$ & $<0,001$ \\
\hline \multicolumn{8}{|l|}{ PROMOCIÓN } \\
\hline Presente & 37,17 & $22,83-51,17$ & 76,71 & $49,65-210,45$ & 82,35 & $49,34-111,19$ & $<0,001$ \\
\hline
\end{tabular}

Nota. * Se estimó el valor $p$ con el test de Kruskal-Wallis para los datos continuos.

Tabla 7. Variables relacionadas con el alcohol por cada 10 ooo habitantes asociadas a los locales de venta de alcohol según características socioeconómicas (NSE) del área en 42 secciones censales de la ciudad de Madrid, 2016.

\begin{tabular}{|c|c|c|c|c|c|c|c|}
\hline & \multicolumn{7}{|c|}{ Características socioeconómicas } \\
\hline & \multicolumn{2}{|c|}{ Bajo (n = 27) } & \multicolumn{2}{|c|}{ Medio ( $n=33$ ) } & \multicolumn{2}{|c|}{ Alto $(n=23)$} & \multirow{2}{*}{ Valor $p^{*}$} \\
\hline & Media & Rango & Media & Rango & Media & Rango & \\
\hline TOTAL LOCALES DE VENTA DE ALCOHOL & 25,87 & $17,06-28,45$ & 25,07 & $15,58-29,61$ & 22,24 & $15,34-32,89$ & 0,776 \\
\hline \multicolumn{8}{|l|}{ DISPONIBILIDAD } \\
\hline \multicolumn{8}{|l|}{ Horario de apertura } \\
\hline Más de 12 horas & 8,48 & $5,17-21,34$ & 10,03 & $6,80-29,03$ & 7,41 & $6,22-27,41$ & 0,448 \\
\hline \multicolumn{8}{|l|}{ PROMOCIÓN } \\
\hline Presente & 16,96 & $7,53-28,45$ & 20,41 & $9,16-29,61$ & 22,24 & $12,35-27,41$ & 0,726 \\
\hline
\end{tabular}

Nota. * Se estimó el valor $p$ con el test de Kruskal-Wallis para los datos continuos. 


\section{Discusión}

Este estudio describe la disponibilidad y promoción de alcohol en locales de venta y/o consumo de alcohol en la ciudad de Madrid, España. Los resultados muestran diferencias en su distribución según tipo de local y NSE del área. Específicamente, encontramos 1) horarios más amplios y mayor presencia de promoción en los locales de venta y consumo que en los locales de venta; y 2) una mayor densidad de locales de venta y/o consumo de alcohol con horarios más amplios y mayor densidad de promoción en las áreas mayor NSE.

\section{Exposición al alcohol en relación a locales de venta y/o consumo de alcohol}

Los resultados muestran una disponibilidad alta de alcohol en la ciudad de Madrid. La densidad media de locales de venta y/o consumo de alcohol en Madrid (56,17 locales por 10000 habitantes) fue más elevada que la densidad de otros lugares como Escocia, Reino Unido (Shortt et al., 2015), Victoria, Australia (Livingston, 2012) o Tallin, Estonia (Orro, Martens, Lepane, Josing y Reinman, 2017). No obstante, fue más baja que la densidad de locales de venta y/o consumo de alcohol identificados en un estudio en la ciudad de Barcelona (España) que usó el mismo instrumento que este estudio (Villalbí et al., 2019).

También encontramos que la mayoría de los locales de venta y/o consumo de alcohol en Madrid tenían un horario amplio de apertura (más de 12 horas), especialmente los locales de venta y consumo. Entre los locales de venta y consumo de alcohol, los bares y establecimientos similares tenían los horarios de apertura más amplios. Recientemente, una directiva de la Unión Europea liberalizó los horarios de apertura de los establecimientos (Anderson y Room, 2011; Villalbí, Bosque-Prous, Gili-Miner, Espelt y Brugal, 2014), por lo que otros países, como Austria y Finlandia, también han ampliado sus horarios de apertura (Organización Mundial de la Salud, 2014). Esta política debería reconsiderarse ya que una mayor facilidad para conseguir alcohol, incluyendo la ampliación de los horarios de venta, estimula el consumo del mismo (Lu, Zhang, Holt, Kanny y Croft, 2018; Trapp, Knuiman, Hooper y Foster, 2018). Las regulaciones sobre la disponibilidad de alcohol son las más efectivas en términos de coste-efectividad para prevenir el consumo de alcohol (Anderson, Chisholm y Fuhr, 2009b). Por tanto, algunas intervenciones deberían considerarse en la agenda para prevenir el consumo de alcohol, como definir una distancia mínima entre locales de venta y/o consumo de alcohol (proximidad entre establecimientos); limitar el número de licencias para la venta o restringir a determinadas tiendas la venta de alcohol (Valiente et al., 2018); aumentar los impuestos sobre el alcohol; o limitar el horario de apertura.

También, observamos que la mayoría de locales de venta y/o consumo de alcohol tenían, como mínimo, un signo de promoción de alcohol. Específicamente, los locales de venta y consumo de alcohol tuvieron una mayor presencia de los mismos, en particular los bares. Una densidad alta de publicidad se ha asociado con un aumento del consumo de la bebida alcohólica promocionada (Kwate y Meyer, 2009; Sillero-Rejon, Maynard e Ibáñez-Zapata, 2020; Westberg et al., 2018) siendo las mujeres y los jóvenes las poblaciones identificadas como las más vulnerables (Kypri, Maclennan, Cousins y Connor, 2018; Ross et al., 2014). Las políticas de la Unión Europea regulan la publicidad de alcohol en medios específicos (i.e., televisión, medios impresos, cine, vallas publicitarias, Internet y redes sociales) (European Alcohol Policy Alliance, 2016). No obstante, la industria del alcohol evade estas políticas promocionando sus marcas en los locales de venta y/o consumo de alcohol, una promoción que pasa desapercibida por muchas personas y que se integra como otro elemento urbano más en las ciudades (Sureda et al., 2017a). La visibilidad continua de bebidas alcohólicas puede aumentar la aceptación y la normalización del consumo de alcohol (Petticrew et al., 2017) y fomentar patrones de consumo de riesgo, especialmente entre jóvenes (Barry et al., 2016). La Organización Mundial de la Salud ha identificado la aceptación social como uno de los nuevos retos para controlar el consumo de alcohol (Organización Mundial de la Salud, 2018), ya que la aceptación se relaciona con patrones de consumo como el binge drinking o el inicio temprano del consumo en jóvenes (Jernigan, Noel, Landon, Thornton y Lobstein, 2017; Lobstein, Landon, Thornton y Jernigan, 2017). Existe suficiente evidencia que apoya la aplicación y ampliación de las regulaciones actuales sobre la publicidad de alcohol. Una alternativa para mejorar la efectividad de estas regulaciones sería implementar intervenciones para controlar el contenido de la publicidad de alcohol (habitualmente vinculada con éxito, deportes y eventos musicales), o el lugar donde se hace esta promoción, prohibiendo cualquier tipo de promoción de alcohol que pudiera verse o percibirse desde la calle (Burton et al., 2017; Chambers et al., 2019).

Estudios previos han encontrado diferencias en cómo los locales de venta y/o consumo de alcohol influyen en el consumo del mismo (Rossheim, Thombs y Suzuki, 2016; Sherk et al., 2018). Nuestros resultados muestran diferencias en la distribución de las características relacionadas con la publicidad de alcohol según los tipos de locales que puede explicar las diferencias halladas en los efectos sobre los patrones de consumo de alcohol. Futuros estudios deberían examinar cómo estas características se relacionan con el consumo y la aceptación del consumo entre las personas expuestas a ellas. La evidencia de estos estudios podría ayudar a diseñar futuras intervenciones en países con un marco político análogo y con tipologías de locales de venta y/o consumo similares. 


\section{Exposición al alcohol en locales de venta y/o consumo según características socioeconómicas del área}

Estudios previos que examinaron cómo la densidad de locales de venta y/o consumo de alcohol difieren según el NSE del área encontraron mayor disponibilidad de locales de venta y/o consumo de alcohol en áreas más deprimidas en términos socioeconómicos (Angus et al., 2017; Rhew et al., 2017). No obstante, en este estudio se encontraron resultados opuestos. En general, la densidad de locales de venta y/o consumo de alcohol y horarios amplios de apertura se encontraron en áreas con un NSE elevado en lugar de en áreas con un NSE bajo. La distribución del uso del suelo en las afueras de la ciudad podría explicar estas diferencias. En ciudades compactas, como Madrid, las áreas con un NSE alto habitualmente se usan por habitantes que se desplazan desde otros barrios por motivos laborales, para hacer compras o con fines de ocio; son lugares céntricos, densamente poblados, con un número elevado de locales de venta y/o consumo de alcohol. Además, los locales de venta y/o consumo de alcohol ubicados en áreas con un NSE alto se relacionan con productos más caros y esto puede afectar a la ubicación del propio local, ya que el poder adquisitivo y la demanda de alcohol de la población garantiza la viabilidad de un negocio relacionado con el alcohol (Schneider y Gruber, 2013). Estas áreas suelen coincidir con zonas turísticas (Veal, 2006). Existe una gran demanda de actividades de ocio alrededor de los lugares turísticos en la ciudad, y esto puede aumentar el número de locales de venta y/o consumo de alcohol, especialmente los de venta y consumo dentro del establecimiento.

Por lo contrario, los locales de venta de alcohol tuvieron la misma distribución según el NSE del área. La gran disponibilidad de locales de venta de alcohol para consumo fuera del establecimiento se ha relacionado con patrones de consumo de riesgo entre jóvenes (Shih et al., 2015; Young et al., 2013). Además, en los locales de venta el alcohol se vende a un precio inferior que en los locales de venta y consumo. El acceso más fácil al alcohol lo hace más atractivo no solo para los jóvenes, sino también para las personas con bajos ingresos. La disponibilidad más alta de locales de venta de alcohol se ha relacionado también con un aumento de signos de consumo de alcohol (Forsyth y Davidson, 2010a). Estos signos incluyen la presencia de basura relacionada con el alcohol y de personas consumiendo alcohol en espacios públicos, a veces en las inmediaciones de los locales de venta de alcohol (Forsyth y Davidson, 2010a; Galloway, Forsyth y Shewan, 2007; Sureda et al., 2017a). Los efectos de la visibilidad de los signos de consumo de alcohol sobre el consumo del mismo son similares a los efectos de la exposición a la publicidad del alcohol (Forsyth y Davidson, 2010b; Villalbí et al., 2019). No incluimos este elemento en el análisis porque la exposición principal en este estudio fueron los locales de venta y/o consumo de alcohol; no obstante, estudios futuros podrían considerar este aspecto del entorno urbano.

La distribución de la publicidad de alcohol asociada a todos los tipos de locales de venta y/o consumo de alcohol y a los locales de venta y consumo de alcohol (considerando cualquier tipo de publicidad) también difirieron, según el NSE del área. La densidad de locales en general (venta y/o consumo de alcohol) y la de locales de venta y consumo de alcohol con presencia de publicidad fue más elevada en áreas con un NSE medio y alto. Estos resultados podrían explicarse por el uso del suelo en estas áreas, y por la mayor densidad de locales de venta y/o consumo de alcohol, especialmente de locales de venta y consumo, que facilitan las oportunidades para promocionar el alcohol.

Estos resultados sugieren una distribución desigual de la disponibilidad y promoción de alcohol en los locales, lo que puede afectar las conductas relacionadas con el alcohol entre las personas expuestas. Estudios previos encontraron una prevalencia mayor de consumo diario de alcohol en áreas con un NSE alto, comparado con áreas deprimidas (Grittner, Kuntsche, Gmel y Bloomfield, 2013; Pabst, Auwera, Piontek, Baumeister y Kraus, 2019). No obstante, patrones de consumo de riesgo como binge drinking tuvieron una prevalencia mayor en zonas más deprimidas (Bellis et al., 2016; Pabst et al., 2019). Reducir la disponibilidad de alcohol mediante normativas que regulen la concesión de licencias y de zonificación han mostrado ser una buena alternativa para disminuir y prevenir estas desigualdades (Hippensteel, Sadler, Milam, Nelson y Furr-Holden, 2018; Jennings et al., 2013). Este tipo de regulaciones identifican las zonas con un exceso de locales de venta y/o consumo de alcohol y los redistribuyen, garantizando distancias mínimas entre ellos. Futuras intervenciones deberían tener en cuenta este tipo de iniciativas para proteger a las personas más expuestas y vulnerables al consumo excesivo de alcohol.

\section{Fortalezas y limitaciones}

Todas las mediciones fueron tomadas entre las 16-21h. Este horario se eligió para garantizar que la mayoría de los locales de venta y/o consumo de alcohol estuviesen abiertos durante la recogida de datos. No obstante, algunos de los pubs y clubs nocturnos estaban cerrados durante la observación. Futuros estudios podrían incluir periodos de observación de 24 horas para captar todos los locales con diferentes horarios de apertura. Aunque no pudimos incluir datos sobre todos los locales de venta y/o consumo de alcohol en Madrid, registramos todos los locales distribuidos en las 42 secciones censales, representativos en términos sociodemográficos y socioeconómicos de la ciudad de Madrid. Tres observadores recopilaron los datos. Recibieron formación de la investigadora principal antes de realizar su trabajo de campo con el fin de reducir la variabilidad inter-observador. Este estudio también presenta fortalezas. La mayoría de los estudios que examinan el 
entorno en relación al alcohol se centran en la disponibilidad de alcohol, medida como la densidad o proximidad de locales de venta y/o consumo de alcohol, y la mayoría se han realizado en Estados Unidos o Australia (Burton et al., 2017; Lu et al., 2018; Trapp et al., 2018). Además, los enfoques utilizados para medir la exposición a la promoción del alcohol no han tenido en cuenta la promoción vinculada a los locales de venta y/o consumo de alcohol, por lo que se subestima la exposición real (Burton et al., 2017; Gentry et al., 2018). Este estudio es el primero en explorar en profundidad la disponibilidad y promoción asociada a locales de venta y/o consumo de alcohol y en comparar sus diferencias y distribución según las características socioeconómicas (NSE) del área. Las diferencias detectadas según el tipo de local de venta y/o consumo de alcohol y en la distribución de las desigualdades sociales reafirman y apoyan la necesidad de que futuros estudios consideren este tipo de análisis y, además, examinen su relación con los patrones de consumo de alcohol.

Este estudio forma parte del proyecto Heart Healthy Hoods (HHH) que tiene como objetivo comprender cómo las características físicas y sociales del entorno urbano pueden afectar la salud cardiovascular. El proyecto $\mathrm{HHH}$ incluye una cohorte de residentes mayores de edad en Madrid; actualmente estamos recogiendo datos sobre sus patrones de consumo de alcohol. Estudios futuros usarán esta información para entender cómo la disponibilidad y promoción de alcohol asociada a los locales de venta y/o consumo de alcohol puede asociarse con los patrones de consumo del mismo.

\section{Conclusiones}

Este es el primer estudio que describe la disponibilidad y promoción asociada a locales de venta y de venta y consumo de alcohol en una muestra representativa de secciones censales de una ciudad grande como es Madrid. Nuestros resultados muestran diferencias en la distribución de la disponibilidad y promoción de alcohol según el tipo de local y el NSE del área. La disponibilidad y promoción de alcohol asociada a los locales de venta y/o consumo de alcohol fue alta, especialmente en los locales de venta y consumo de alcohol. Además, la distribución de la disponibilidad y promoción de alcohol varió según el NSE del área. Hubo mayor disponibilidad y promoción de alcohol en las áreas con un alto NSE, en comparación con áreas con un bajo NSE.

Han de darse pasos en el futuro para endurecer las normativas que regulan la disponibilidad y promoción de alcohol asociada a los locales de venta y de venta y consumo de alcohol considerando las desigualdades socioeconómicas en la ciudad.

\section{Reconocimientos}

X.S. y A.P. tuvieron la idea original. X.S. supervisó la recogida de datos. A.P. preparó las bases de datos y analizó los datos, según las recomendaciones de X.S. y A.E. A.P. y X.S. redactaron el manuscrito. Todos los autores contribuyeron de manera sustancial en la interpretación de los datos, la revisión del manuscrito y la aprobación de su versión definitiva. X.S. y M.F. son los investigadores principales. Los autores desean expresar su gratitud a Irene Molina por participar en la recogida de datos.

\section{Conflicto de intereses y ayudas económicas}

Este estudio recibió apoyo del Plan Nacional sobre Drogas (Ayuda n. ${ }^{\circ} 2016 \mathrm{I} 047$ y n. ${ }^{\circ} 2016 \mathrm{I} 029$ ). El proyecto Heart Healthy Hoods recibió financiación del Consejo Europeo de Investigación bajo el Séptimo Programa Marco de la Comunidad Europea (FP7/2007-2013/ERC Starting Grant Heart Healthy Hoods Agreement n. ${ }^{\circ} 623$ 336893). El Grupo de Investigación de Control del Tabaquismo recibe apoyo de la Agencia de Gestión de Ayudas Universitarias y de Investigación de la Generalitat de Catalunya [2017SGR319]. Las fuentes de financiación no han influido en el del diseño del estudio; la recogida, el análisis o la interpretación de los datos; la redacción del manuscrito; ni en la decisión de presentar el manuscrito para su publicación. Los autores declaran la inexistencia de conflictos de interés. Albert Espelt es editor asociado de la revista Adicciones, pero no asumió papel alguno en el proceso de publicación.

\section{Referencias}

Anderson, P., Chisholm, D. y Fuhr, D. C. (2009b). Effectiveness and cost-effectiveness of policies and programmes to reduce the harm caused by alcohol. The Lancet, 373, 2234-2246. doi:10.1016/S01406736(09)60744-3.

Anderson, P., De Bruijn, A., Angus, K., Gordon, R. y Hastings, G. (2009a). Impact of alcohol advertising and media exposure on adolescent alcohol use: A systematic review of longitudinal studies. Alcohol and Alcoholism, 44, 229-243. doi:10.1093/alcalc/agn115.

Anderson, P. y Room, R. (2011). Addictions and European policy: Has the 'European project' stifled science-led policy? Drug and Alcohol Review, 30, 117-118. doi:10.1111/ j.1465-3362.2010.00269.x.

Angus, C., Holmes, J., Maheswaran, R., Green, M. A., Meier, P. S., Brennan, A.,... Anderson, P. (2017). Mapping patterns and trends in the spatial availability of alcohol using low-level geographic data: A case study in England 2003-2013. International Journal of Environmental Research and Public Health, 14, 406. doi:10.3390/ijerph14040406. 
Barry, A. E., Bates, A. M., Olusanya, O., Vinal, C. E., Martin, E., Peoples, J. E.,... Montano, J. R. (2016). Alcohol marketing on Twitter and Instagram: Evidence of directly advertising to youth/adolescents. Alcohol and Alcoholism, 51, 487-492. doi:10.1093/alcalc/agv128.

Bellis, M. A., Hughes, K., Nicholls, J., Sheron, N., Gilmore, I. y Jones, L. (2016). The alcohol harm paradox: Using a national survey to explore how alcohol may disproportionately impact health in deprived individuals. BMC Public Health, 16, 111. doi:10.1186/ s12889-016-2766-x.

Bosque-Prous, M., Espelt, A., Guitart, A. M., Bartroli, M., Villalbí, J. R. y Brugal, M. T. (2014). Association between stricter alcohol advertising regulations and lower hazardous drinking across European countries. Addiction, 109, 1634-1643. doi:10.1111/add.12562.

Bryden, A., Roberts, B., Petticrew, M. y McKee, M. (2013). A systematic review of the influence of community level social factors on alcohol use. Health E Place, 21, 70-85. doi:10.1016/j.healthplace.2013.01.012.

Burton, R., Henn, C., Lavoie, D., O’Connor, R., Perkins, C., Sweeney, K.,... Sheron, N. (2017). A rapid evidence review of the effectiveness and cost-effectiveness of alcohol control policies: An English perspective. The Lancet, 389, 1558-1580. doi:10.1016/S01406736(16)32420-5.

Chambers, T. I. M., Stanley, J., Pearson, A. L., Smith, M., Barr, M., Mhurchu, C. N. I. y Signal, L. (2019). Quantifying children's non-supermarket exposure to alcohol marketing via product packaging using wearable cameras. Journal of Studies on Alcohol and Drugs, 80, 158166. doi:10.15288/jsad.2019.80.158.

Costa, D. A. da S., Mingoti, S. A., Andrade, A. C. de S., Xavier, C. C., Proietti, F. A. y Caiaffa, W. (2017). Indicators of physical and social neighborhood attributes measured by the Systematic Social Observation method. Cadernos de Saúde Pública, 33, e00026316. doi:10.1590/0102$311 x 00026316$.

Cunradi, C. B. (2010). Neighborhoods, alcohol outlets and intimate partner violence: Addressing research gaps in explanatory mechanisms. International Journal of Environmental Research and Public Health, 7, 799-813. doi:10.3390/ijerph7030799.

Esser, M. B., Waters, H., Smart, M. y Jernigan, D. H. (2016). Impact of Maryland's 2011 alcohol sales tax increase on alcoholic beverage sales. The American Journal of Drug and Alcohol Abuse, 42, 404-411. doi:10.3109/00952990.2 016.1150485 .

European Alcohol Policy Alliance. (2016). European Report on Alcohol Policy. Recuperado de https:// www.eurocare.org/media/GENERAL/docs/reports/ 2016europeanreportonalcoholpolicy.pdf.

Forsyth, A. J. M. y Davidson, N. (2010a). Community offsales provision and the presence of alcohol-related detritus in residential neighbourhoods. Health $\mathcal{E}$ Place, 16, 349-358. doi:10.1016/j.healthplace.2009.11.003.

Forsyth, A. J. M. y Davidson, N. (2010b). The nature and extent of illegal drug and alcohol-related litter in Scottish social housing community: A photographic investigation. Addiction Research $\mathcal{E}$ Theory, 18, 71-83. doi:10.3109/16066350802559464.

Galán, I., Valencia-Martín, J. L., Guallar-Castillón, P. y Rodríguez-Artalejo, F. (2014). Alcohol drinking patterns and biomarkers of coronary risk in the Spanish population. Nutrition, Metabolism, and Cardiovascular Diseases, 24, 189-197. doi:10.1016/j.numecd.2013.07.002.

Galloway, J., Forsyth, A. J. M. y Shewan, D. (2007). Young people's street drinking behaviour: Investigating the influence of marketing \& subculture. Glasgow: United Kingdom. Recuperado de http://www.sccjr.ac.uk/wpcontent/uploads/2008/11/Young_Peoples_Street_ Drinking_Behaviour.pdf.

Gentry, E., Poirier, K., Wilkinson, T., Nhean, S., Nyborn, J. y Siegel, M. (2018). Alcohol advertising at Boston subway stations: An assessment of exposure by race and socioeconomic status. Australian and New Zealand Journal of Public Health, 42, 120-130. doi:10.2105/AJPH.

Giesbrecht, N., Huguet, N., Ogden, L., Kaplan, M. S., McFarland, B. H., Caetano, R., ... Nolte, K. B. (2015). Acute alcohol use among suicide decedents in 14 US states: impacts of off-premise and on-premise alcohol outlet density. Addiction, 110, 300-307. doi:10.1111/ add.12762.

Griswold, M. G., Fullman, N., Hawley, C., Arian, N., Zimsen, S. R. M., Tymeson, H. D., ... Gakidou, E. (2018). Alcohol use and burden for 195 countries and territories, 1990-2016: A systematic analysis for the Global Burden of Disease Study 2016. The Lancet, 392, 1015-1035. doi:10.1016/S0140-6736(18)31310-2.

Grittner, U., Kuntsche, S., Gmel, G. y Bloomfield, K. (2013). Alcohol consumption and social inequality at the individual and country levels-results from an international study. European Journal of Public Health, 23, 332-339. doi:10.1093/eurpub/cks044.

Gullón, P., Bilal, U., Cebrecos, A., Badland, H. M., Galán, I. y Franco, M. (2017). Intersection of neighborhood dynamics and socioeconomic status in small-area walkability: the Heart Healthy Hoods project. International Journal of Health Geographics, 16, 16-21. doi:10.1186/s12942-017-0095-7.

Hackbarth, D. P., Silvestri, B. y Cosper, W. (1995). Tobacco and alcohol billboards in 50 Chicago neighborhoods: Market segmentation to sell dangerous products to the poor. Journal of Public Health Policy, 16, 213-230. doi:10.2307/3342593.

Halonen, J. I., Kivimäki, M., Virtanen, M., Pentti, J., Subramanian, S. V., Kawachi, I. y Vahtera, J. (2013). Proximity of off-premise alcohol outlets and heavy 
alcohol consumption: A cohort study. Drug and Alcohol Dependence, 132, 295-300. doi:10.1016/J. DRUGALCDEP.2013.02.022.

Hay, G. C., Whigham, P. A., Kypri, K. y Langley, J. D. (2009). Neighbourhood deprivation and access to alcohol outlets: A national study. Health E Place, 15, 1086-1093. doi:10.1016/j.healthplace.2009.05.008.

Hippensteel, C. L., Sadler, R. C., Milam, A. J., Nelson, V. y Furr-Holden, D. M. (2018). Using zoning as a Public Health tool to reduce oversaturation of alcohol outlets: An examination of the effects of the new "300 Foot Rule" on packaged goods stores in a Mid-Atlantic city. Prevention Science, 20, 833-843. doi:10.1007/s11121-018-0947-9.

Jennings, J. M., Milam, A. J., Greiner, A., Furr-Holden, D. M., Curriero, F. C. y Thorton, R. J. (2013). Neighborhood alcohol outlets and the association with violent crime in one Mid-Atlantic city: The implications for zoning policy. Journal of Urban Health, 91, 62-71. doi:10.1007/ s11524-013-9821-z.

Jernigan, D., Noel, J., Landon, J., Thornton, N. y Lobstein, T. (2017). Alcohol marketing and youth alcohol consumption: A systematic review of longitudinal studies published since 2008. Addiction, 112, 7-20. doi:10.1111/ add.13591.

Kwate, N. y Meyer, I. (2009). Association between residential exposure to outdoor alcohol advertising and problem drinking among African American women in New York City. American Journal of Public Health, 99, 228230. doi:10.2105/AJPH.2007.132217.

Kypri, K., Maclennan, B., Cousins, K. y Connor, J. (2018). Hazardous drinking among students over a decade of university policy change: Controlled before-and-after evaluation. International Journal of Environmental Research and Public Health, 15, 2137. doi:10.3390/ijerph15102137.

Lee, W. N. y Callcott, M. F. (1994). Billboard advertising: A comparison of vice products across ethnic groups. Journal of Business Research, 30, 85-94. doi:10.1016/01482963(94)90071-X.

Livingston, M. (2012). The social gradient of alcohol availability in Victoria, Australia. Australian and New Zealand Journal of Public Health, 36, 41-47. doi:10.1111/ j.1753-6405.2011.00776.x.

Lobstein, T., Landon, J., Thornton, N. y Jernigan, D. (2017). The commercial use of digital media to market alcohol products: A narrative review. Addiction, 112, 2127. doi:10.1111/add.13493.

Lu, H., Zhang, X., Holt, J. B., Kanny, D. y Croft, J. B. (2018). Quantifying spatial accessibility in public health practice and research: An application to on-premise alcohol outlets, United States, 2013. International Journal of Health Geographics, 17, 1-13. doi:10.1186/s12942-0180143-y.

Mair, C., Gruenewald, P. J., Ponicki, W. R. y Remer, L. (2013). Varying impacts of alcohol outlet densities on violent assaults: Explaining differences across neighborhoods. Journal of Studies on Alcohol and Drugs, 74, 50-58. doi:10.15288/jsad.2013.74.50.

Major, J. M., Sargent, J. D., Graubard, B. I., Carlos, H. A., Hollenbeck, A. R., Altekruse, S. F.,.. McGlynn, K. A. (2014). Local geographic variation in chronic liver disease and hepatocellular carcinoma: Contributions of socioeconomic deprivation, alcohol retail outlets, and lifestyle. Annals of Epidemiology, 24, 104-110. doi:10.1016/j.annepidem.2013.11.006.

Morrison, C., Gruenewald, P. J. y Ponicki, W. R. (2015). Socioeconomic determinants of exposure to alcohol outlets. Journal of Studies on Alcohol and Drugs, 76, 439446. doi:10.15288/jsad.2015.76.439.

Organización Mundial de la Salud. (2014). European status report on alcohol and health 2014. Geneva: Switzerland. Recuperado de http://www.euro.who.int/_data/ assets / pdf_file/0019/244900/Marketing-of-alcoholicbeverages.pdf.

Organización Mundial de la Salud. (2018). Global Status report on alcohol and health 2018. Geneva: Switzerland. Recuperado de https://www.who.int/substance_abuse/ publications/global_alcohol_report/gsr_2018/en/.

Orro, E., Martens, K., Lepane, L., Josing, M. y Reinman, M. (2017). Alcohol market, consumption and harms in Estonia.Tallin: Estonia. Recuperado de https://dea. digar.ee/cgi-bin/dea?a=d\&d=JValkoturg201811.2.9.

Pabst, A., Auwera, S., Piontek, D., Baumeister, S. E. y Kraus, L. (2019). Decomposing social inequalities in alcohol consumption in Germany 1995 - 2015: An age-periodcohort analysis. Addiction, 114, 1359-1368. doi:10.1111/ add.14616.

Petticrew, M., Shemilt, I., Lorenc, T., Marteau, T. M., Melendez-Torres, G. J., O'Mara-Eves, A.,... Thomas, J. (2017). Alcohol advertising and public health: Systems perspectives versus narrow perspectives. Journal of Epidemiology and Community Health, 71, 308-312. doi:10.1136/jech-2016-207644.

Plan Nacional sobre Drogas. (2017). Encuesta sobre alcohol y otras drogas en España (EDADES 2017). Recuperado de http://www.pnsd.mscbs.gob.es/profesionales/ sistemasInformacion/sistemaInformacion/pdf/EDADES_2017_Informe.pdf.

Popova, S., Giesbrecht, N., Bekmuradov, D. y Patra, J. (2009). Hours and days of sale and density of alcohol outlets: Impacts on alcohol consumption and damage: A systematic review. Alcohol and Alcoholism, 44, 500-516. doi:10.1093/alcalc/agp054.

Raudenbush, S. W. y Sampson, R. J. (1999). Toward a science of assessing ecological settings, with application to the systematic social observation of neighborhoods. Sociological Methodology, 29, 1-41. doi:10.1111/00811750.00059 . 
Rhew, I. C., Kosterman, R. y Lee, J. O. (2017). Neighborhood typologies associated with alcohol use among adults in their 30s: A finite mixture modeling approach. Journal of Urban Health, 94, 542-548. doi:10.1007/s11524-017-01612.

Richardson, E. A., Hill, S., Mitchell, R., Pearce, J. R. y Shortt, N. K. (2015). Is local alcohol outlet density related to alcohol-related morbidity and mortality in Scottish cities? Health Eं Place, 33, 172-180. doi:10.1016/j. healthplace.2015.02.014.

Ross, C. S., Maple, E., Siegel, M., Dejong, W., Naimi, T. S., Padon, A. A.,... Jernigan, D. H. (2014). The relationship between population-level exposure to alcohol advertising on television and brand-specific consumption among underage youth in the US. Alcohol and Alcoholism, 50, 358-364. doi:10.1093/alcalc/agv016.

Rossheim, M. E., Thombs, D. 1. y Suzuki, S. (2016). Association between alcohol outlets and HIV prevalence in U.S. counties. Journal of Studies on Alcohol and Drugs, 77, 898-903. doi:10.15288/jsad.2016.77.898.

Rowland, B., Evans-Whipp, T., Hemphill, S., Leung, R., Livingston, M. y Toumbourou, J. W. (2016). The density of alcohol outlets and adolescent alcohol consumption: An Australian longitudinal analysis. Health E Place, 37, 43-49. doi:10.1016/j.healthplace.2015.11.004.

Rowland, B., Toumbourou, J. W., Satyen, L., Tooley, G. A., Hall, J., Livingston, M. y Williams, J. (2014). Associations between alcohol outlet densities and adolescent alcohol consumption: A study in Australian students. Addictive Behaviors, 39, 282-288. doi:10.1016/j. addbeh.2013.10.001.

Schneider, S. y Gruber, J. (2013). Neighbourhood deprivation and outlet density for tobacco, alcohol and fast food: First hints of obesogenic and addictive environments in Germany. Public Health Nutrition, 16, 1168-1177. doi:10.1017/S1368980012003321.

Scribner, R. A., Cohen, D. A. y Fisher, W. (2000). Evidence of a structural effect for alcohol outlet density: A multilevel analysis. Alcoholism: Clinical and Experimental Research, 24, 188-195. doi:0145-6008/00/2402-0188\$03.00/.

Sherk, A., Stockwell, T., Chikritzhs, T., Andréasson, S., Angus, C., Gripenberg, J.,... Woods, J. (2018). Alcohol consumption and the physical availability of take-away alcohol: Systematic reviews and meta-analyses of the days and hours of sale and outlet density. Journal of Studies on Alcohol and Drugs, 79, 58-67. doi:10.15288/ jsad.2018.79.58.

Shih, R. A., Mullins, L., Ewing, B. A., Miyashiro, L., Tucker, J. S., Pedersen, E. R.,... D 'amico, E. J. (2015). Associations between neighborhood alcohol availability and young adolescent alcohol use. Psychology of Addictive Behaviors, 29, 950-959. doi:10.1037/adb0000081.

Shortt, N. K., Tisch, C., Pearce, J. R., Mitchell, R., Richardson, E. A., Hill, S. y Collin, J. (2015). A cross-sectional analysis of the relationship between tobacco and alcohol outlet density and neighbourhood deprivation. BMC Public Health, 15, 1014. doi:10.1186/s12889-015-2321-1.

Sillero-Rejon, C., Maynard, O. e Ibáñez-Zapata, J.Á. (2020). Atención visual hacia el etiquetado de bebidas alcohólicas: Un estudio exploratorio basado en eye-tracking. Adicciones, 32, 202-207. doi:10.20882/ adicciones.1207.

Sudhinaraset, M., Wigglesworth, C., y Takeuchi, D. T. (2015). Social and cultural contexts of alcohol use. Alcohol Research: Current Reviews, 38, 35-45.

Sureda, X., Bilal, U., Fernández, E., Valiente, R., Escobar, F. J., Navas-Acien, A. y Franco, M. (2018b). Secondhand smoke exposure in outdoor hospitality venues: Smoking visibility and assessment of airborne markers. Environmental Research, 165, 220-227. doi:10.1016/j. envres.2018.04.024.

Sureda, X., Carreño, V., Espelt, A., Villalbí, J. R., Pearce, J. R. y Franco, M. (2018a). Alcohol in the city: Wherever and whenever. Gaceta Sanitaria, 32, 172-175. doi:10.1016/j. jeurceramsoc.2011.03.031.

Sureda, X., Espelt, A., Villalbí, J. R., Cebrecos, A., Baranda, L., Pearce, J. R. y Franco, M. (2017b). Development and evaluation of the OHCITIES instrument: Assesing alcohol urban environments in the Heart Healthy Hoods project. BMJ Open, 7, e017362. doi:10.1136/ bmjopen-2017-017362.

Sureda, X., Villalbí, J. R., Espelt, A., y Franco, M. (2017a). Living under the influence: Normalisation of alcohol consumption in our cities. Gaceta Sanitaria, 31, 66-68. doi:10.1016/j.gaceta.2016.07.018.

Trapp, G. S. A., Knuiman, M., Hooper, P. y Foster, S. (2018). Proximity to liquor stores and adolescent alcohol intake: A prospective study. American Journal of Preventive Medicine, 54, 825-830. doi:10.1016/j. amepre.2018.01.043.

Valiente, R., Sureda, X., Bilal, U., Navas-Acien, A., Pearce, J., Franco, M. y Escobar, F. (2018). Regulating the local availability of tobacco retailing in Madrid, Spain: A GIS study to evaluate compliance. Tobacco Control, 28, 325333. doi:10.1136/tobaccocontrol-2018-054269.

Veal, A. J. (2006). Research methods for leisure and tourism: A practical guide (Pearson Education, ed.).

Villalbí, J. R., Bosque-Prous, M., Gili-Miner, M., Espelt, A. y Brugal, M. T. (2014). Políticas para prevenir los daños causados por el alcohol. Revista Española de Salud Pública, 88, 515-528. doi:10.4321/S1135-57272014000400006.

Villalbí, J. R., Espelt, A., Sureda, X., Bosque-Prous, M., Teixidó-Compañó, E., Puigcorbé, S.,... Brugal, M. T. (2019). The urban environment of alcohol: A study on the availabiblity, promotion and visibility of its use in the neighborhoods of Barcelona. Adicciones, 31, 33-40. doi:10.20882/adicciones.950. 
Waller, M. W. e Iritani, B. J. (2013). Violence victimization of young men in heterosexual relationships: Does alcohol outlet density influence outcomes? Violence and Victims, 27, 527-547. doi:10.1891/0886-6708.27.4.527.

Wechsler, H., Lee, J. E., Hall, J., Wagenaar, A. C. y Lee, H. (2002). Secondhand effects of student alcohol use reported by neighbors of colleges: The role of alcohol outlets. Social Science E Medicine, 55, 425-435. doi:10.1016/S0277-9536(01)00259-3.

Westberg, K., Stavros, C., Smith, A. C. T., Munro, G. y Argus, K. (2018). An examination of how alcohol brands use sport to engage consumers on social media. Drug and Alcohol Review, 37, 28-35. doi:10.1111/dar.12493.

Young, R., Macdonald, L. y Ellaway, A. (2013). Associations between proximity and density of local alcohol outlets and alcohol use among Scottish adolescents. Health E Place, 19, 124-130. doi:10.1016/j.healthplace.2012.10.004. 